\title{
STIMULI PRODUCED BY LIGHT AND BY CONTACT WITH SOLID WALLS AS FACTORS IN THE BEHAVIOR OF OPHIUROIDS ${ }^{1}$
}

\author{
R. P. COWLES \\ Associate in Biology, Johns Hopkins University
}

THIRTEEN FIGURES.

The work of Preyer (1886-1887) on the reactions of echinoderms, in which he ascribed intelligence to these lowly creatures and saw in their behavior a true "will," has stimulated many to study the behavior of starfish, ophiuroids and sea-urchins. While all investigators since his time undoubtedly recognize the excellence of Preyer's observations and experiments, none have had the courage to endow echinoderms with intelligent action. Some, such as Romanes and Ewart (1881), deny the existence of any psychic quality in these animals; others, such as Loeb (1900), Glaser (1907) and Jennings (1907), who have repeated some of Preyer's experiments see no intelligence indicated, while such as von Uexküll (1905), and Bohn (1908), and probably some of the above-mentioned workers also, will neither deny nor affirm its existence, although their experiments do not lead them to believe that echinoderms possess intelligence. Observation and experiment up to the present time do not confirm Preyer's contention and we have no cases indicating associative memory among the echinoderms, yet Jennings (1907), Bohn (1908), and Cole (1910), have shown that starfishes form habits.

${ }^{1}$ It gives me pleasure to thank the Carnegie Institution of Washington for the privilege of working in their marine biological laboratory. I also wish to express my appreciation of the courtesy and aid extended to me by Dr. Alf red G. Mayer, the director of the laboratory. 
The beautiful coördination in the movements of the parts of the starfish has always been a subject which has occupied the minds of those who have observed the movements of echinoderms; we find nearly all who have studied the reactions of the starfish or sea-urchin offering observations, experiments and hypotheses to explain the harmonious working of the tube-feet. If one turns a starfish over so that it lies with its oral side uppermost for a longer or shorter time, the tube-feet wave about in an uncoördinated manner, but finally a time is reached when one sees a definite tendency for the tube-feet to move together seemingly in the interest of righting the animal. What is it that governs this sudden organized movement? To Preyer the phenomenon seems to be regulated by the nerve ring which acts as a centre; similarly, Romanes and Ewart recognized a coördinating centre as in part responsible for the action; von Uexküll, who seems to find no coördinating centre in the sea-urchin, considers coördination in the latter to be due to the action of one part on another; Loeb rules out the central nerve ring and also even ganglia as coördination centres, and judging from his work on the Medusae would probably consider the coördination as due to the simple facts of irritability and conductivity of the colloidal substances of the tissues, thus making the problem one of physical chemistry; to Jennings it is a physiological problem based on individual history and racial development; and finally to the vitalists the problem deals with a determining factor which is non-mechanical. We still seem to be as far from the solution as were Romanes, Ewart and Preyer.

During the last few years there has been much work done on the study of the reactions of the lower forms to photic, chemical, mechanical and electrical stimuli. Most investigators have paid especial attention and have laid especial stress on the uniformity in the response to a given stimulus. Jennings, however, both in his work on the lower organisms and also in his study of the starfish, has placed in the foreground the somewhat neglected idea that there is much variability in the reactions of organisms to a given stimulus; that differences in physiological states often cause differences in the reaction to this given stimulus. However, all who have studied the echinoderms have recognized the variabil- 
ity in reaction. Preyer, Romanes, Ewart and von Uexküll speak of it, and Glaser looks upon the ophiuroid as "versatile." Bohn, whose paper on the reactions of starfishes and ophiuroids appeared shortly after that of Jennings on the starfish, recog. nizes variability in reaction and the influence of physiological states; he believes, however, and rightly, too, that certain external stimuli may be so strong as to annihilate any manifestations of internal states.

It is the purpose of this paper to record some observations made on ophiuroids under natural conditions and to describe some experiments undertaken in the laboratory to test the influence on movement, of light stimuli and stimuli produced by contact with solid walls. Incidentally, some observations and experiments are included dealing with the climbing of vertical walls and with the reactions to food. While many of the experiments made to test the effect of light and contact stimuli are of such a character as to produce considerable uniformity in the reaction to a given stimulus, and while it has been the writer's purpose to show the rather definite influence of these stimuli, yet every experiment and every observation has indicated that the behavior of the ophiuroid is not stereotyped. At times, when an ophiuroid is subjected to a great difference in light intensity, the reaction seems to the casual observer to be stereotyped; but this is not the case, for every now and then the behavior is such as to upset all calculations on the part of the observer. Given a certain definite external stimulus acting on an ophiuroid, one cannot always predict the resulting behavior correctly.

Several species of brittle-stars are quite abundant among the coral reefs and coral rocks in the region surrounding Loggerhead Key, Florida, and the clearness of the sea-water makes it easily possible to observe these animals in their natural habitat. The well-equipped marine laboratory of the Carnegie Institution, with its large aquaria and "live cars," affords the investigator an excellent opportunity to experiment with them.

There are seven or eight species of brittle-stars which are commonly found about Loggerhead Key. Of these, the large Ophiocoma riisei was used as a rule in the following experiments, 
although Ophiocoma echinata and Ophiura appressa were frequently experimented with for comparison.

In order to keep the brittle-stars in the best condition possible for work in the laboratory, they were placed in large open "live cars," in which were pieces of coral and rock to afford them shelter. These "live cars" were floated in the open sea and were so arranged that there was a constant circulation of pure sea-water. Ophiuroids are extremely sensitive to impure water and they soon show the effects of the same, so care was taken when working with them to change the water frequently in order to prevent any unusual behavior.

\section{LOCOMOTION}

There seems to be a very general agreement, either definitely stated or implied, that in the case of ophiuroids no one ray has a greater functional value in locomotion than another, although, so far as I know, no careful statistical study has been made. Preyer (1886-1887), Grave (1900), Glaser (1907), Bohn (1908.)2

In other words, it seems probable that a normal ophiuroid may use any ray or interradius as a director, and apparently in the long run does not use one ray or interradius more than another. There are times, however, during experiments and even under natural conditions when an ophiuroid may use a certain ray or interradius as director exclusively. This behavior can usually be traced to the lasting effect of some previous stimulus, such as contact with a more or less vertical surface or to some stimulus such as difference in light intensity.

\section{TUBE-FEET AS LOCOMOTOR ORGANS}

The statement that the ambulacral appendages of the ophiuroids do not act as locomotor organs can no longer be accepted since the studies of Grave (1900) on Ophiura brevispina and von Hj. Östergren (1904) oๆ Ophiocoma nigra.

\footnotetext{
2 Bohn, however, finds that sometimes large specimens of the starfish, Asterias rubens, show "une sorte de préférence pour certain bras" (p. 29); Cole (1910), as a result of his statistical study of the locomotion of the starfish, finds that there is a tendency to use certain rays more than others.
} 
My observations on the ophiuroids found among the coral reefs near Loggerhead Key confirm the observations of these two investigators and show that these appendages are important both in climbing and feeding. The ambulacral appendages of Ophiocoma riisei seem to be quite well developed when compared with those of some other ophiuroids, although they are not so serviceable in locomotion as those of the starfish. Grave (1900) has found that Ophiura brevispina uses the tube-feet in ordinary locomotion by fitting them into the irregularities of the surface, thus forming hold-fasts for the rest of the arm to pull against. The same behavior was observed in Ophiocoma riisei, but as in the case of Ophiura brevispina, when an individual moves fast it does so by the stroke movement of the rays and the ambulacral appendages do not seem to aid. Jennings (1907) has shown that in the starfish, A sterias forreri de Loriol, the appendages are used in a somewhat similar manner to the human leg. This is probably true for all starfishes, but only to a small extent for the ophiuroids. When an individual moves slowly such movements of the tube-feet may be seen but they are not very important in locomotion.

Until the publication of the observations of von $\mathrm{Hj}$. Östergren (1904) on the climbing of Ophiocoma nigra it was generally supposed that the brittle-stars did not use their tube-feet in a sucker-like manner. My study of Ophiocoma riisei shows that while this form has no definite suckers, such as we find at the end of the tube-feet of starfishes and sea-urchins, yet its tube-feet have an adhesive function which is brought into play principally while the creatures are climbing up rocks or other vertical surfaces. If a specimen of Ophiocoma riisei is placed with its oral surface against the side of a glass aquarium filled with sea-water and held there for a few seconds until the tube-feet can be applied to the glass the animal will fasten itself and remain for some time. Close examination with a lens will show that many of the ambulacral appendages have their tips closely pressed against the glass, and that often when they are drawn away they loosen their hold with a little jerk, showing that something was holding them. No adhesive matter could be found at the ends of the tube-feet nor 
could any mucous glands be seen in their tissues. While there are no definite suckers present, it is undoubtedly true that a temporary sucker is formed when the foot presses iteslf against the glass.

It is not only when the ophiuroid is held against the side of the aquarium that it adheres, for it often does so of its own accord, climbing up almost to the surface of the water. This occurs usually when there are no rocks or crevices for shelter at the bottom of the aquarium. I have observed similar behavior in the case of Ophiocoma echinata. Ophiura appressa will alsoadhere if it is held for a time against the glass but neither of these two species is so well adapted in this direction as Ophiocoma riisei.

It might be claimed that the ophiuroids which climb the sides of the aquarium are "positively phototactic" or "positively photopathic," but this can hardly be true because the ophiuroids are well known for their tendency to move into places where the light is very dim We might also say that they are "negatively geotropic;' but if we mean by this that they are compelled to move up the sides of the aquarium on account of the stimulus produced by the attraction of gravitation, I do not believe that we have used the correct term. On the contrary, I believe that Ophiocoma riisei as the result of certain stimuli such as difference in light intensity or unfavorable conditions either external or internal, forms the impulse to move in a certain direction; having reached the wall of the aquarium it will continue moving forward and in so doing mount the wall unless the conditions of contact alter the impulse when it may crawl along the base of the wall.

\section{TUBE-FEET AS FEEDING ORGANS}

Ophiocoma riisei sometimes takes its food as von Uexküll (1904) describes; that is, by a twisting of the arm which progresses from the point at which the food particle touches the tube-feet to the region of the mouth, but in most cases the food particle (e.g., piece of fresh fish) is taken by the tube-feet and carried along by them until it reaches the mouth. 
Hamann (1900) and Cuenot (1891) state that the tube-feet are taste organs or organs of smell, while Preyer (1886-1887) and Nagel (1894) believe the tentacles or tube-feet in the region of the mouth have the same function.

While watching the feeding of Ophiocoma I became interested in testing the tube-feet in this respect. In order to see how the ophiuroid reacted to what was food and what was not, a small pellet of paper was used instead of the particle of fish. This was placed close to a ray and, as a rule, it was picked up by the tube-feet, carried along for about two or three centimeters and then dropped. When a small piece of fish was substituted for the pellet of paper it was as a rule carried the full length of the ray to the mouth. This seems to show that the ophiuroid reacted to food without bringing it to the mouth although of course the evidence is not conclusive.

Several series of tests were made with rays amputated about one centimeter from the disc and placed with their oral i.e., tubefeet surface uppermost. A small piece of fish placed close to the tip of the ray was taken up at once by the tube-feet and carried in a quite normal manner to the cut end of the ray where it was dropped. When, however, a small pellet of wet paper was used, although it was taken up at once, it was not carried to the proximal end of the ray, but was simply rolled about through a distance of a centimeter or two and then dropped.

It would occur to anyone that in the test just described we might be dealing with the tactile sense, and that the consistency of the fish and paper might have something to do with the result, so the following test was made. Two pellets of wet paper of approximately the same size and consistency were prepared. One of these was dipped in sea-water in which a piece of fresh fish had been soaking and the other was merely dipped in sea-water. A ray which had been amputated an hour previously was then tested with these two pellets, as in the above experiment. The pellet without fish flavor was taken up by the tube-feet and almost immediately rejected, while the one with the fish juices on it was seized and carried rapidly to the cut end of the ray. A repetition of these tests at intervals of five or ten minutes gave the same results as those just described. 
These experiments undoubtedly show that the rays react to food even as far out as the tip and independently of the mouth or the rest of the disc. Furthermore, they indicate that sense organs are present either on the tube-feet or on the ambulacral surface of the rays, which are sensitive to organic substances in solution or suspension and that some of these substances stimulate the tube-feet either directly or indirectly in such a manner that objects are carried toward the mouth, while others do not.

\section{METHOD OF RIGHTING}

The method of righting of ophiuroids has been studied by Grave (1900), von Uexküll (1904) and Glaser (1907). Grave (p. 87) says: "Two adjacent arms straighten out so that together they form a straight line. On these arms as an axis the body revolves, being pushed over by the three remaining arms, but mostly by the median one of the three." Von Uexküll (p. 11) finds that when Ophioglypha is placed on its aboral surface, the rays become bent under so as to raise the disc off the bottom and the weight of the disc causes the ophiuroid to topple over into its normal position. Glaser (p. 208) recognizes a factor in the mechanics of turning which I think is quite important. He says that movements occur at the bases of the straightened arms and in the interradial portion of the disc between them whose effect is to start the righting.

In fresh, healthy specimens of Ophiocoma echinata, which have not been tired out by experiments and which have been kept in pure sea-water, the behavior in righting is as follows: As soon as the specimen is placed on its aboral side the tube-feet begin to move in all directions, and almost at the same time the disc is raised off the bottom by the stiffening and pushing of the rays. Then two of the rays spread out much as an acrobat "doing the split," their distal ends twist over into their normal position and the disc begins to turn over. Usually the leverage seems to be at the proximal end of the rays near where they are attached to the disc (i.e., the two rays forming "the split"). There seems to be a decided twisting as a result of the contraction of certain 
muscles of the rays which are attached to the somewhat rigid skeleton. This twisting starts the turning over of the disc which, after a certain point, continues as the result of its own weight, drawing the other rays over after it. The tube-feet do not aid in the righting nor do the three rays which are drawn over help as a usual thing. In fact, often very active specimens seem to right themselves without raising up the disc but simply by twisting the proximal parts of the two rays forming "the split." It must be remembered that Ophiocoma echinata and Ophiocoma riisei have comparatively long and heavy rays.

In order to test the assumption that the twisting of the rays is an important point in righting, three of the rays were amputated close to the disc of a specimen which was then placed in a dish of sea-water with the ambulacral surface uppermost. Almost immediately the two remaining rays assumed "the split" like position, the distal ends turned over and the twisting continued until the disk was brought into its normal position. This series of movements was accomplished with apparently little difficulty and the specimen was even successful in righting itself when some pressure was applied to the edge of the inverted disc from which the rays were amputated.

Four rays were removed from another individual, which was then inverted and in a similar manner by the twisting of a single ray the disc was righted.

These experiments seem to show quite clearly that the twisting of the rays is an important part of the righting of Ophiocoma echinata and that the righting can be accomplished by the movements of the two of the rays without the aid of the other three.

\section{BEHAVIOR UNDER NATURAL CONDITIONS}

Before discussing the experiments carried on in the laboratory to test the behavior toward light and other factors, I shall describe some observations made on ophiuroids in their natural habitat. One of the best places to find these creatures, in the region around Loggerhead Key, is on the western side of a typical coral reef lying a short distance off the east side of Bird Key. 
Part of this reef is exposed at low tide and part is not. Under the light rocks of the unexposed part many ophiuroids live. If one of these rocks is turned over, in the majority of cases, several specimens are brought to light, and among these one usually finds Ophiocoma riisei and Ophiura appressa.

It is well known that ophiuroids are sensitive to light. Some of them (Ophiocoma riisei) seem to be very sensitive, while others, such as Ophiolepis ciliata, which Bohn (1908) studied, are not so sensitive. In general, however, I think it may be said that ophiuroids react negatively to brightly lighted fields unless some other factor changes the reaction. Now let us see what occurs when a rock, covering ophiuroids, is overturned on a bright day. As a usual thing it will be found that they move in the direction toward which the stone has been turned without reference to the position of the sun. This does not mean that they are not sensitive to brightly lighted regions but that there are some other factors which determine the direction of movement. The ophiuroid usually has one or more rays touching the under surface of the rock or twisted around some projection, and the stimulation produced by this contact is retained by these rays so that they move forward with these rays in advance in the direction the stone has been moved. In other words, they "show memory of past stimulation" as Jennings (1907) calls it. The stimulus of contact with some raised solid surface, such as the side of a rock, is retained for some little time as we shall see in experiments to be described later. Even a small stone lying on the bottom often has an effect on a moving ophiuroid when a ray touches it; the contact is very apt to cause hesitancy when the creature is moving in a certain direction even though the stone affords practically no shadow and no shelter.

The following behavior also shows the importance of contact stimuli in the movements of ophiuroids. If a specimen which is lying under the shelter of a rock with one or more rays against it is drawn out with the hand, keeping the rays in the same general position with reference to the shelter, the ophiuroid will usually return to about the same place if it is not removed to too great a distance. This behavior is apparently not a reaction to the shade 
of the rock, for if the specimen is turned around while being taken away from the rock it will usually move with the ray or rays directed forward, which were originally against the rock, even though this behavior may take the ophiuroid directly away from the shelter. The influence of handling by the investigator at once suggests itself as a factor in the behavior just described. In fact, experiments below show that echinoderms do tend to move away from the side of the body which has been handled, but the explanation of the behavior given above nevertheless holds good, as is shown by changing the manner of holding.

\section{CONTACT WITH SOLID WALLS}

It has been mentioned above that stimuli produced by contact with solid walls, such as the surface of rocks, are very important factors in the life of the ophiuroid. Experiments which will be described below indicate that they are even more important than stimuli produced by small differences in intensity of light.

The following rather crude experiment shows the lasting effect of a stimulus produced by contact with the solid walls of the corner of a rectangular glass dish. This dish, $36 \mathrm{~cm}$. long, 23.5 $\mathrm{cm}$. wide and $7.5 \mathrm{~cm}$. high, was partly filled with sea-water and placed in front of a wide-open window. Light from many sources entered the dish but that from the open window was of the greatest intensity. An ophiuroid (Ophiocoma riisei) was placed in the dish and it moved at once to the corner indicated, (Fig. 1, a) placing one of its rays in the angle. After allowing the specimen to remain there for several minutes, its was pushed diagonally across the dish to the position shown (fig. 1,b). The orientation of the ophiuroid with reference to the original resting place was not changed during this procedure and its rays were not allowed to touch the sides or corners of the dish. Almost immediately the creature moved back to the corner from which it had been removed, (Fig. 1, $a$ ) even though in so doing it went toward the strongest source of light. It did not turn the disc but moved with the ray forward that had originally been in contact with the corner. This experiment was repeated many times, using different corners for the starting point, and in nearly every case the ophiur- 
oid returned to the corner in which it had been resting, although sometimes, especially when fatigued, the return was not so direct as that shown in fig. 1.

One naturally asks if the behavior just described is due to the persistence of the contact-stimulus? May not bilateral symmetry,

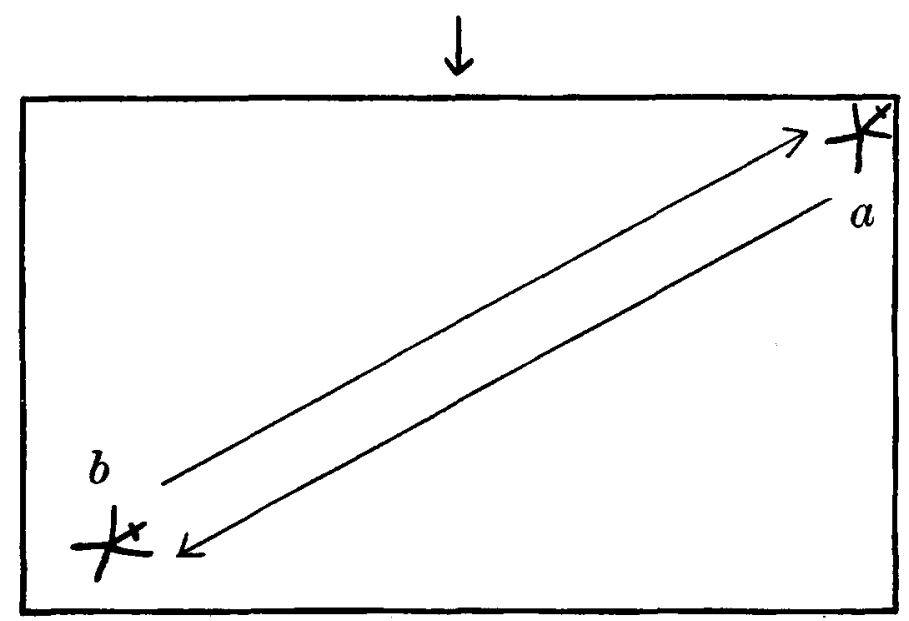

\section{EXPLANATION OF FIGURES}

The large rectangle in the thirteen figures of this article representes the outline of the large rectangular glass dish; the arrow outside of the rectangle indicates the direction from which the most intense light comes; the plain arrows inside the rectangle show the direction of locomotion; the arrows with hooks on the end indicate that the ophiuroid has been turned on its back or that it has righted itself; the ophiuroid is represented by a five armed star and in any one figure it is always the same arm or ray that is marked by a short cross line.

method of handling, intensity of the lighted field, or currents in the water, due to pushing the ophiuroid away from its resting place be factors?

In order to see if bilateral symmetry or any structural peculiarity were factors in the above behavior, many trials were made in 
which each different ray was subjected to the stimulus produced by the corner contact. There is no bilateral symmetry evident on the surface of $O$. riisei, so track was kept of the different rays used simply by watching them closely and also by marking the rays with a small loop of thread. The results showed conclusively that no one ray had any greater functional value than another.

While there is no doubt that the method of taking hold of an ophiuroid may have an effect on its behavior, yet by varying this

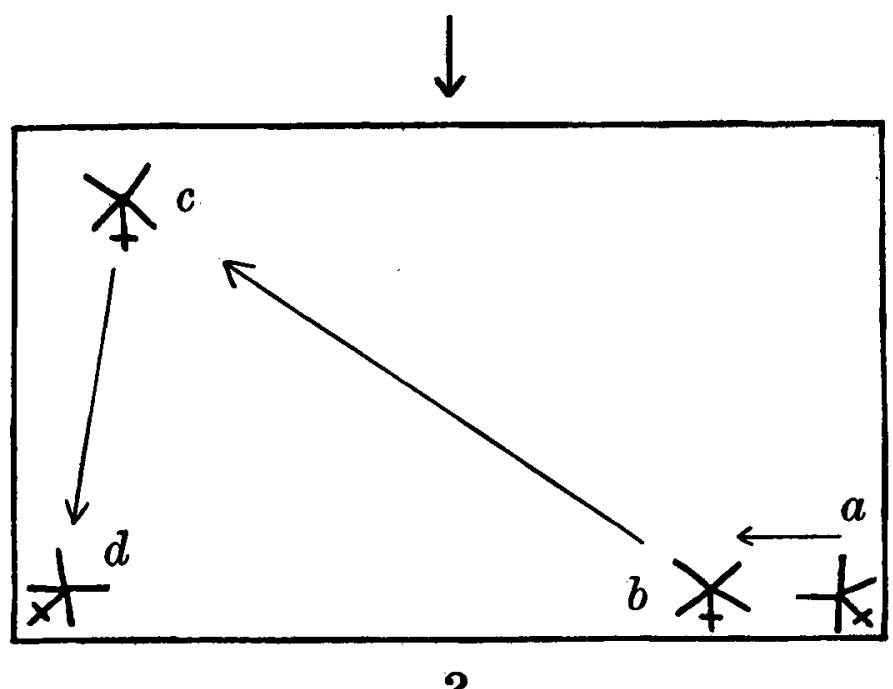

2

method it was found that the behavior under consideration was not due to the handling.

Further to test the persistence of this contact stimulus, the following two experiments were tried. A specimen with a ray resting in the corner (fig. $2 a$ ) was turned so that this ray was directed as shown at $b$ and then pushed to the position $c$. This specimen then instead of returning to position $a$ or $b$ moved to the position $d$. That is, it moved with the ray forward that had originally been in contact with the corner at $a$.

In another experiment an individual resting at $a$ (fig. 3 ) was turned through $180^{\circ}(b)$, then pushed to position $c$. It then moved 
directly to the corner $d$, with the ray leading that had been in the corner at $a$.

These two experiments show again in quite a clear manner the persistence of the stimulus produced by the contact or breaking of the contact of the ray with the corner of the dish.

The current in the water produced by pushing the ophiuroid from one region to another seems to have no effect on the behavior. If, for example, we pick up the ophiuroid and carry it out of the water to another position in the dish the behavior is the same as when the specimen is pushed through the water. An experiment was tried in which an ophiuroid was taken from the corner in which it was resting (fig. $4, a$ ), and was then carried around the room as indicated. It was placed at $b$ and immediately began to move with the marked ray as director toward its original resting place, but finally turned and stopped in the corner $c$. The behavior here was not quite so characteristic as usual, but the tendency to use the ray that had been stimulated was evident. Similar trials in which the test was less complicated gave much more accurate results.

I have described only a few experiments of over a hundred that were tried, and I do not wish to leave the impression that the results were always as definite as those mentioned or that there were no other factors operating during the experiments; but one cannot avoid the conclusion that the stimulus produced by the contact or breaking of contact of the ray with a solid wall is a very important factor in the behavior of the ophiuroid. The effect of this stimulus often persists for some little time, but it may easily disappear and in fact be completely dominated by large differences in the intensity of light.

\section{CONTACT STIMULI AND LIGHT STIMULI}

In order to compare the effects of contact stimuli due to solid walls and the stimuli due to dimly lighted regions, many series of experiments were tried. Their relative value in determining the direction of locomotion was tested. 

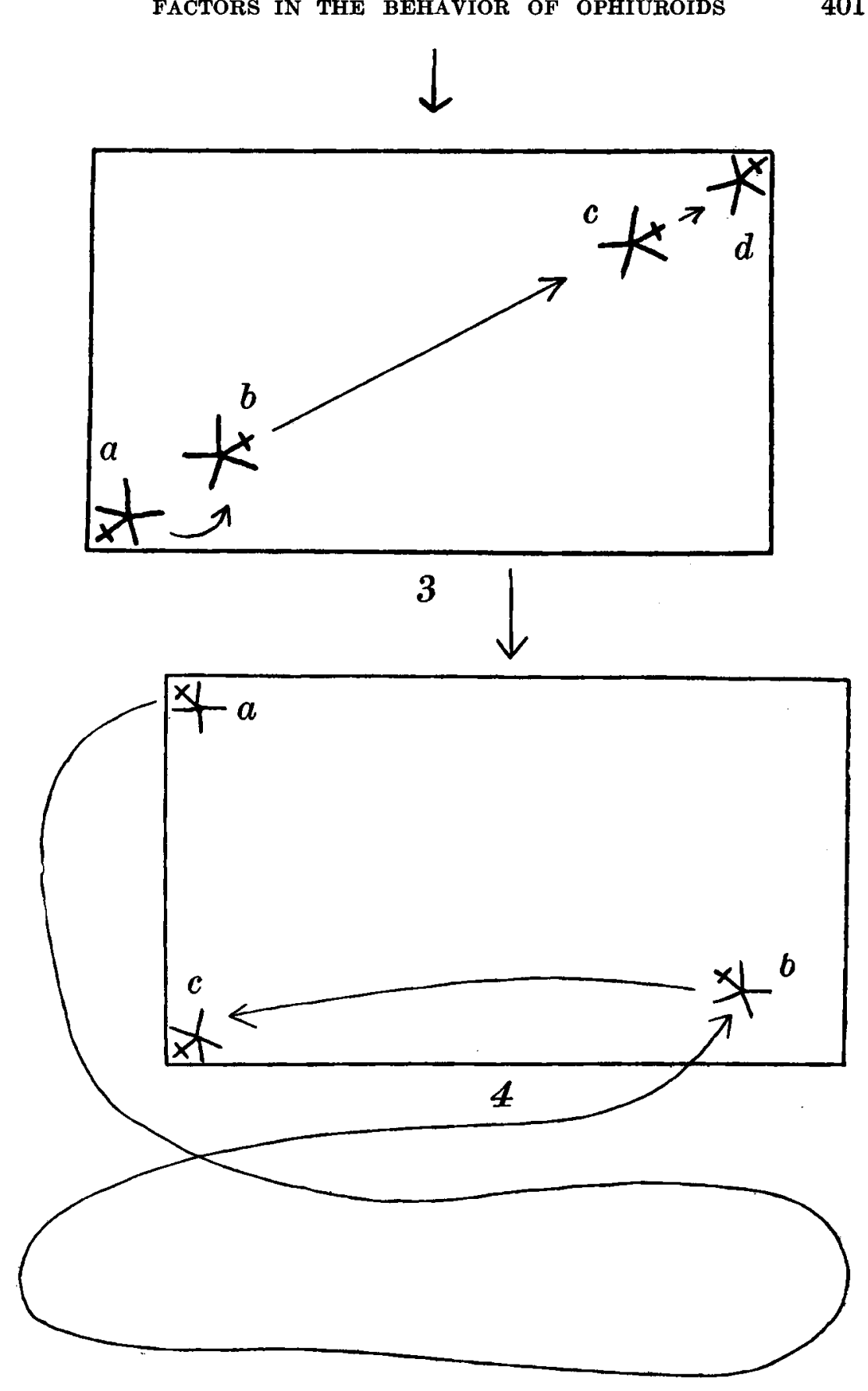
We shall consider only one of these series which is a characteristic one. In the first place an ophiuroid wassubmitted to several trials such as have been described above and it was found that the behavior was as usual. That is, the contact stimulus in the corner of the glass dish persisted as a rule and seemed to be the determining factor in the specimen's return when, without changing its orientation, it was pushed away. Then the corner in which the ophiuroid was resting was shaded by placing dark screens around it. As one would naturally expect, since it is known that ophiuroids react positively to dimly lighted regions, the specimen moved in the usual manner when put to trial (fig. 5). That is, after being drawn out from the darkened corner, without change of orientation, it returned again, with the contact ray directed forward. The behavior on reaching its resting place was somewhat different from that exhibited in the preceding experiments. The ophiuroid assumed a more settled attitude; it curled its rays up to some extent, thus occupying a smaller space.

After several trials the conditions were changed (fig. 6) by also placing dark screens around the corner, diagonally opposite to the one originally shaded. The ophiuroid was then pushed to the position $b$. After some hesitation, undoubtedly due to the new shaded region, it moved to $c$, but without touching the corner walls, went to $d$ and finally back to $a$, assuming its original attitude with the same ray in the corner. The behavior seemed to indicate a conflict between two guiding stimuli; the shaded region apparently had an influence, for the specimen changed its usual direction of movement and went into the shaded pocket $c$, using the interradius opposite to the contact ray as director. Almost immediately, however, it came out again and moved by the way of $d$ to its original resting place with the contact ray leading, apparently still under the influence of the contact stimulus received at $a$.

The experiment just described was repeated seven times, first using one shaded corner as the starting point and then the other shaded corner. In every case but one the behavior was the same, except that the specimen remained in the shaded corner into which it had moved (fig. 7). The ophiuroid moved to the shaded 

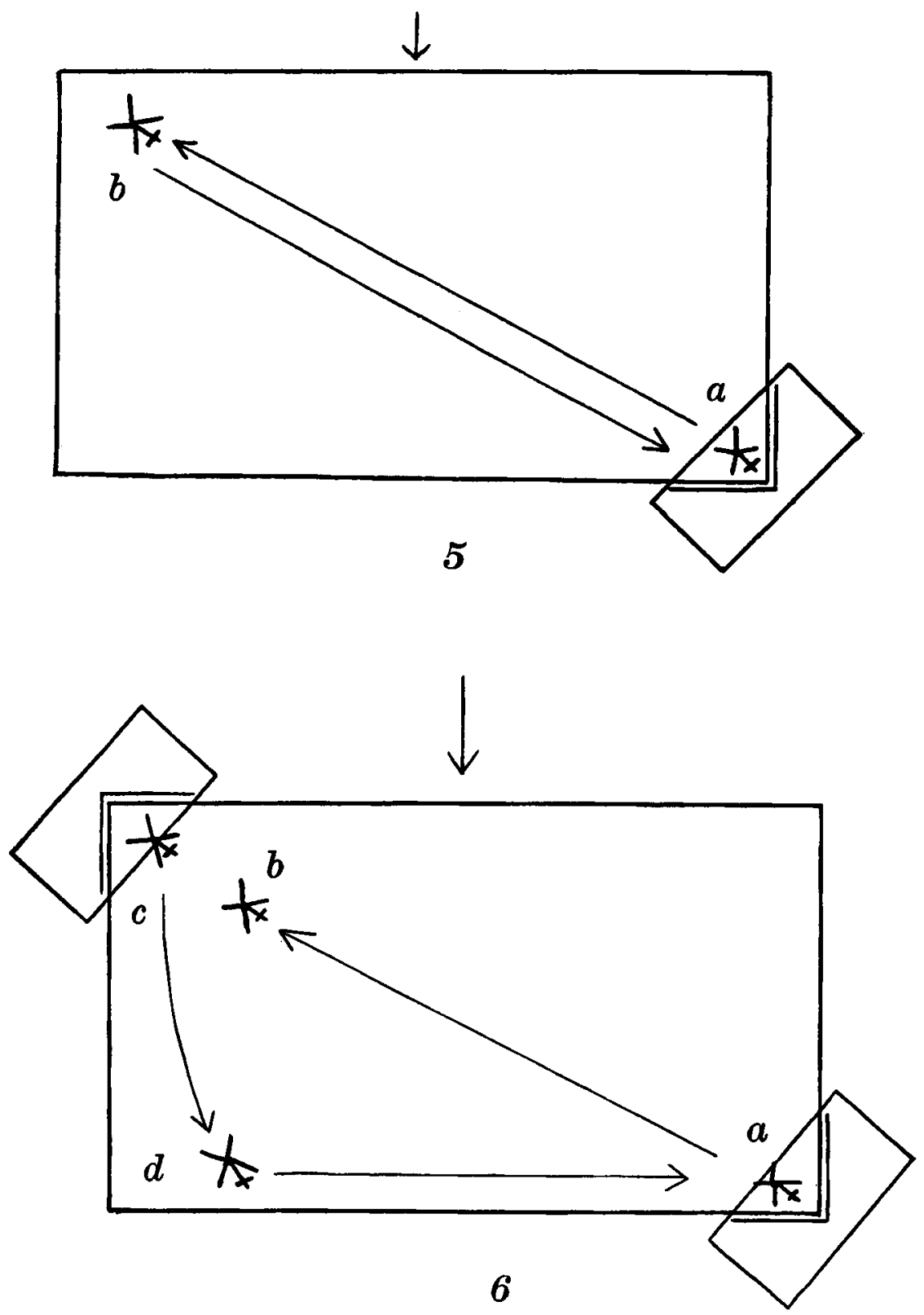
region opposite to its original resting place; that is, apparently without reference to the contact stimulus. In other words, the stimulus produced by the proximity of a shaded region seemed to dominate over the stimulus produced by contact with the corner walls.

The above series of experiments was finally completed by submitting the ophiuroid to a number of trials similar to those described at the beginning of the series. That is, the screens were removed from both corners and the specimen was tested in respect to the effect of the contact stimulus. Its behavior now became similar to that at the beginning of the series. The ophiuroid reacted definitely to the stimulus produced by contact or breaking of contact with the corner walls of the dish. It must be mentioned, however, that the return to the starting point was not so accurate, a fact which may be due to a persisting effect of the stimulus of the darkened regions or to fatigue.

From these experiments we may draw the following conclusions: Solid walls and darkened regions are important factors in the behavior of Ophiocoma riisei, Ophiocoma echinata and Ophiura appressa. The stimulus produced by the contact or breaking of contact with solid walls, especially a corner, persists for an appreciable time, long enough so that the ophiuroid will react to the stimulus some little time after and will tend to return to the object producing it, or more accurately, will tend to move with the ray or rays that received the stimulus forward. A region of reduced light intensity, such as the dark pocket in the above experiments, also acts as a stimulus on these ophiuroids and produces a positive reaction. The darkened region seems to have an effect from some little distance (several centimeters) a phenomenon observed by Jennings (1907) for the starfish and by Bohn (1908) while testing the effect of light and dark screens on ophiuroids. This phenomenon will be discussed later. An analysis of the relative value of these two factors in the behavior seems to show that the stimulus produced by a region of much reduced light intensity is more important than the contact stimulus in determining the direction of locomotion. However, when these ophiuroids move into a darkened region the subsequent behavior is dependent on 
FACTORS IN THE BEHAVIOR OF OPHIUROIDS

405
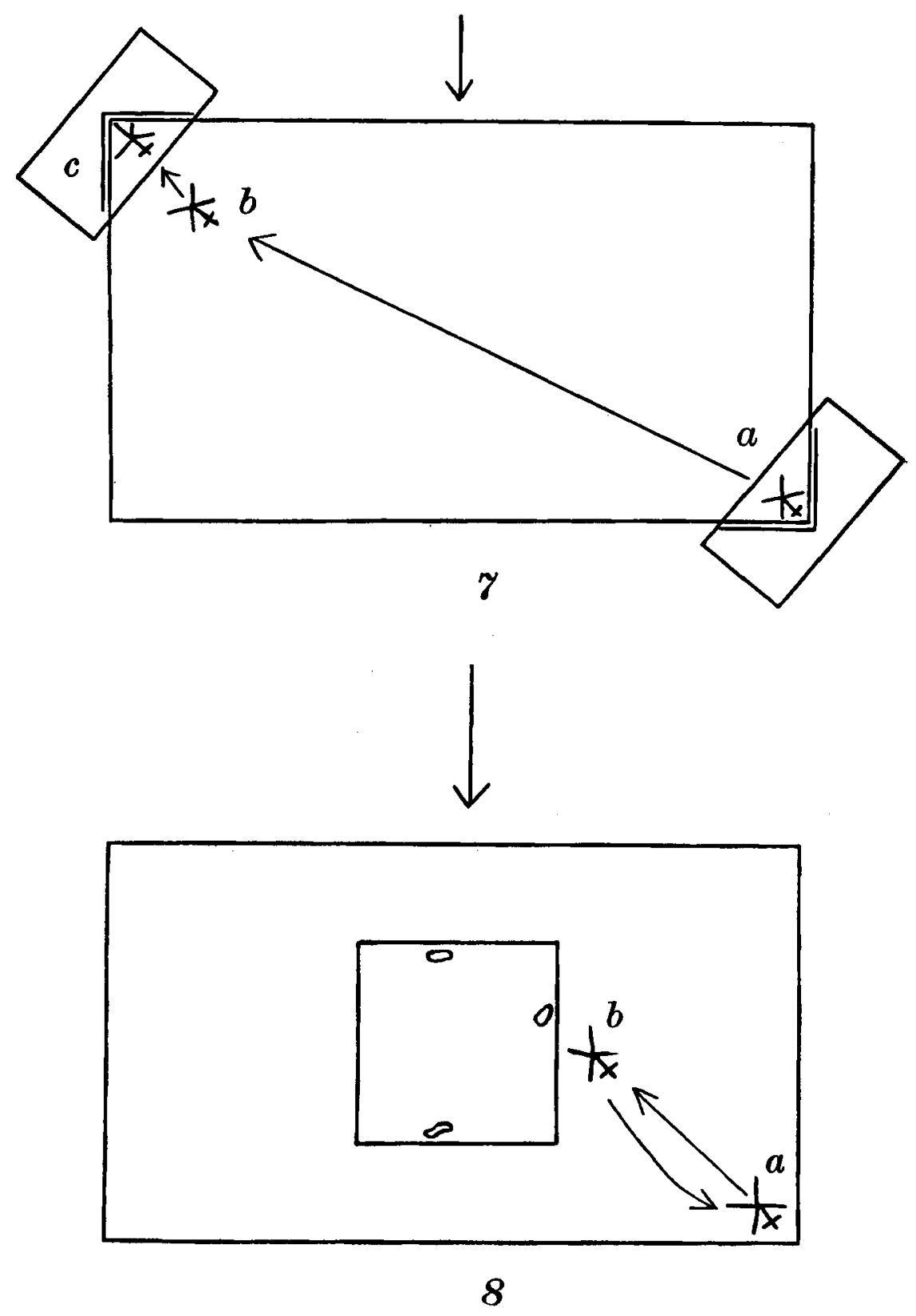
whether there are solid objects such as stones or walls, against which they may put their rays. That is, they will not, as a rule, remain in the darkened region unless such objects are present.

\section{EFFECT OF HORIZONTAL DARK SCREENS}

In order to determine the effect of darkened regions with or without the stimuli produced by contact with solid walls, a series of experiments were made, in which a region of shade was produced in the middle of the experimenting dish by supporting a piece of black glass in the water horizontally by means of three pieces of coral (fig. 8). In this way a shadow was cast on the bottom of the dish without forming a pocket, such as that in the experiments described above. A region of reduced light intensity was produced in the field without introducing any dark vertical walls.

Many trials were made similar to that shown in fig. 8 , in which the ophiuroid was removed from its resting place $a$ and pushed to the position $b$, close to the shaded region. In no case did the specimen pass under the screen, the behavior thus differing from the experiments described above, in which there was a dark pocket. This difference may have been due to the greater light intensity of the field in the former case than in the latter, but I believe that the important factor was the absence of vertical dark walls, which seem to have an effect, as Jennings (1907) and Bohn (1908) have shown.

After the series of trials just described, another series was tried, in which the black screen was suspended in the water in a horizontal position about two centimeters from the bottom without using any pieces of coral for support. Again and again an ophiuroid was tested in the manner shown in fig. 8, and also in fig. 9 . In the former case the specimen did not move under the shaded region, and in the latter, although it passed under it, it never remained, but returned to the original brightly-lighted resting place, unless by chance one of its rays touched the hanging screen, when it stayed under, although its rays did not remain in contact 

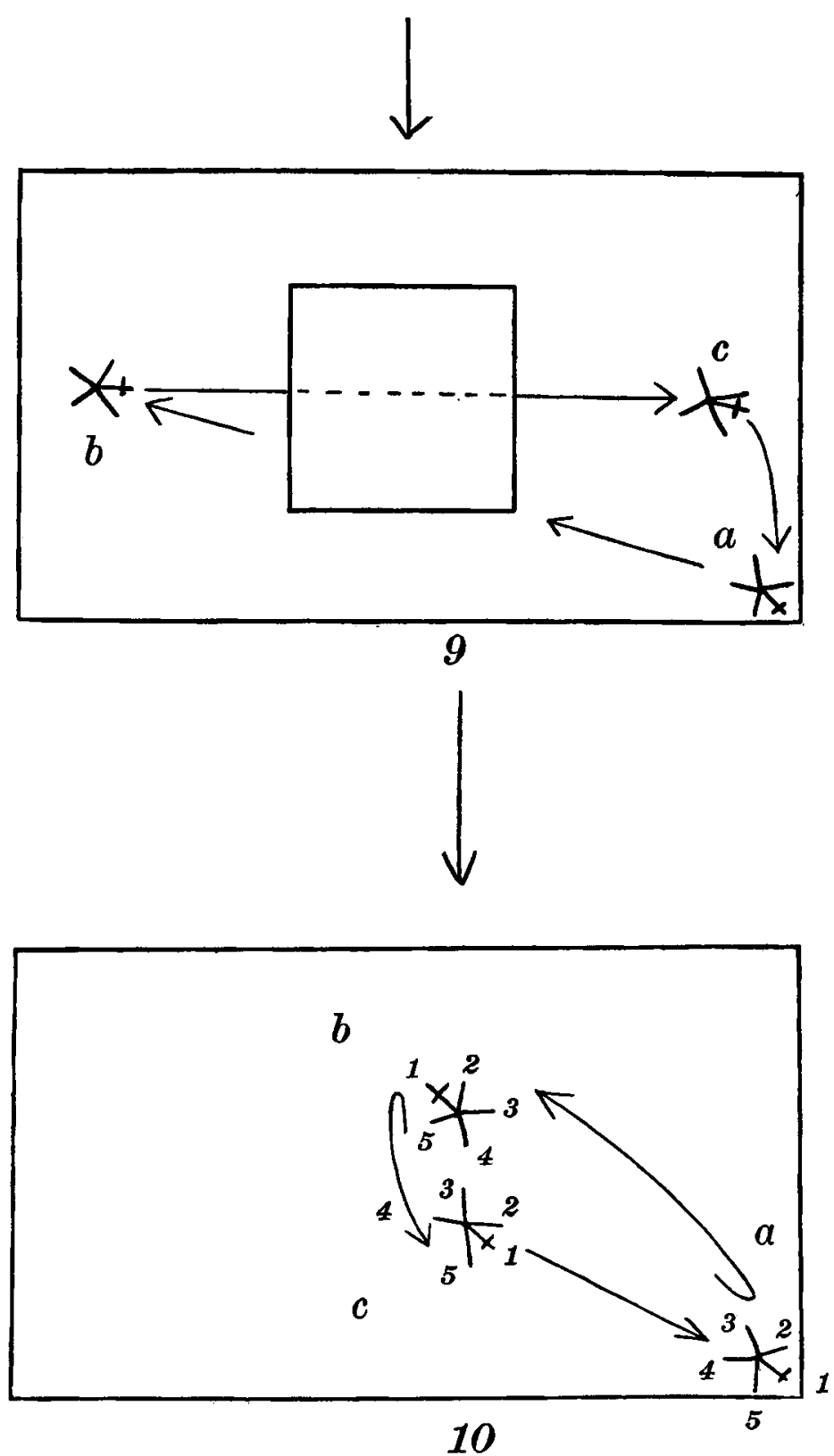
with the latter. If a piece of coral was placed under the horizontal screen the behavior of the ophiuroid was almost invaciably changed.

Instead of moving through the shaded region, the specimen put one or more of its rays against the coral, tilted its disc and remained quiet as though the conditions for its comfort were satisfied.

\section{EFFECT OF LIGHT AND CONTACT STIMULI ON DIRECTION OF RIGHTING}

The ophiuroids with which I have worked do not seem to show any permanent habit of using a certain pair of rays on which to right themselves. That is, when one of the creatures with rays of about equal length is inverted in a dish of water, care being taken to rule out light, handling, etc., no one pair seems to have greater functional value than another. Normal structural characters do not seem to determine the rays used, but when such external factors as light, contact with solid walls and handling are allowed to operate, they seem to be of much importance in determining the direction of righting and consequently the rays used. Undoubtedly internal physiological conditions do at times influence the behavior, but there does not seem to be a permanent habit of righting on a certain pair of rays persisting from day to day.

In the series of experiments from which the following typical cases are taken, the same glass dish filled with sea-water was placed in front of a wide window as in the previous experiment, so that the strongest light came from the side indicated by the straight arrow in the figures. An ophiuroid was placed in the water and allowed to come to rest with one of its rays in the corner $a$. The rays were then numbered, as shown in fig. 10, ray 1 being the ray in contact with the corner. When the ophiuroid was taken from the position $a$, inverted and placed at the position $b$, so that the ray 1 was pointed in a diametrically opposite direction, the creature righted itself on rays 3 and 4, returning directly to its original position $a$. Similar trials were made many times, and as a general rule the behavior was the same as that just described. Now the 


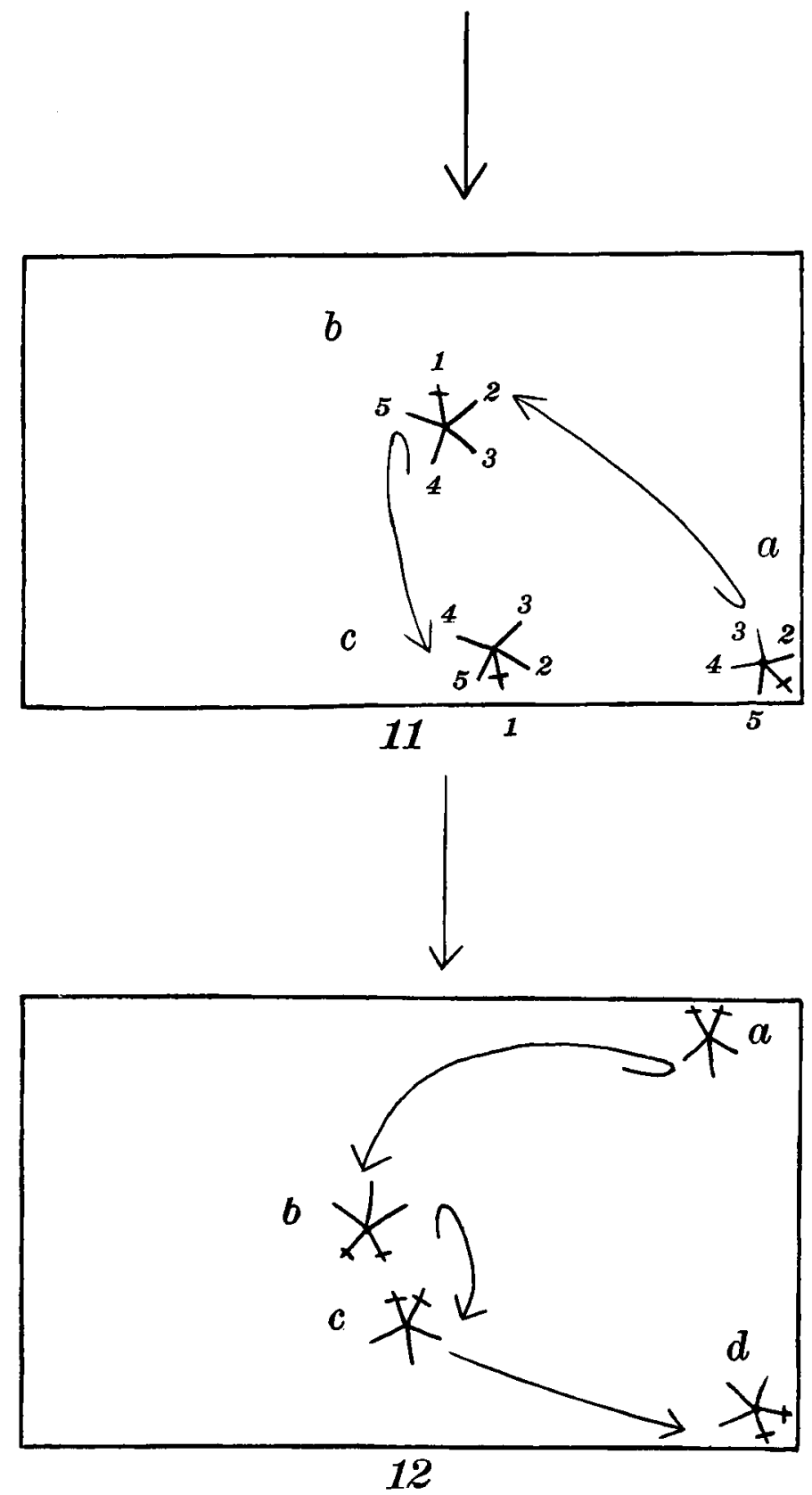




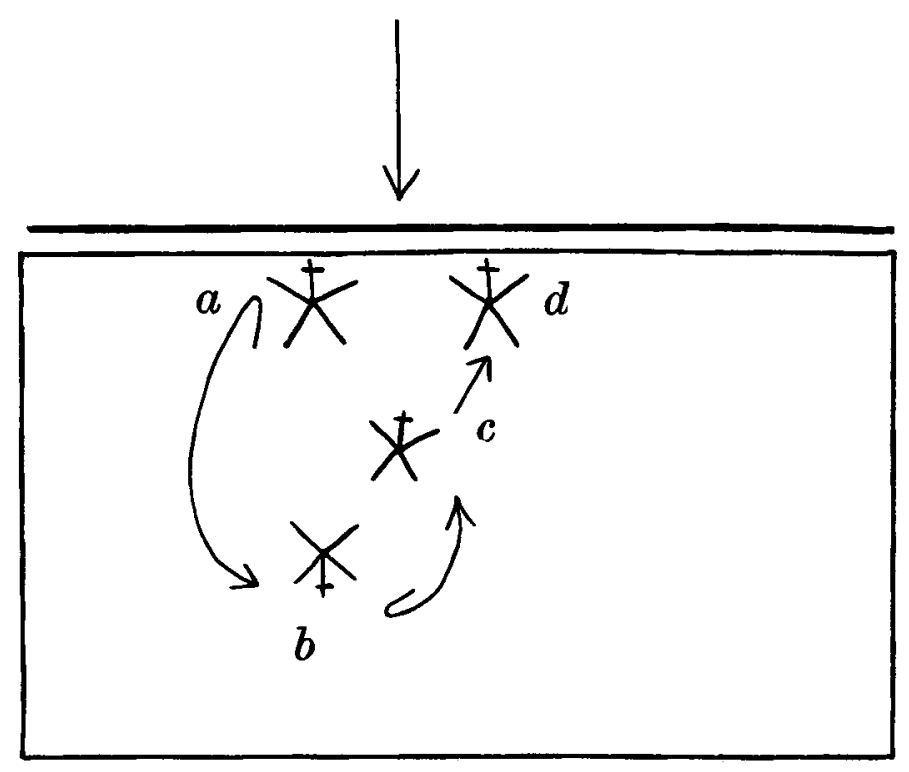

question arises: Are we dealing here with the effect of light stimuli or with the effect of stimuli produced by contact with the wall of the dish or with both?

The following experiment among many others shows that the stimulus due to contact is to be reckoned with; in other words, that the stimulus produced by the contact of ray 1 with the solid walls of the corner of the dish or the stimulus produced by the breaking of this contact has an effect on the direction of righting, on the rays employed in this righting, and on the direction of locomotion directly after the completion of the act. An ophiuroid (fig. 11) resting at $a$ against the corner walls was inverted and placed at $b$, so that ray 1 was not directed in a diametrically opposite direction to that in position $a$. The result was that the ophiuroid righted itself on rays 3 and 4 , and then moved, with ray 1 as the directing ray, apparently under the influence of the stimulus given to ray 1 when it was resting in the corner $a$. For 
this reason, it would, seem the specimen did not return to its original resting place.

While the contact stimulus received before the ophiuroid is inverted surely is a factor in determining the direction of righting, the intensity of the light is undoubtedly a more important one. It certainly plays an important part in the two experiments just described. Both factors are responsible for the behavior observed, for when rimilar trials were made, in which the stimulus of contact with the corner walls was such as would tend to make an ophiuroid right itself toward the bright light of the window, the creature did not, as a rule, right itself in that direction. Such a result is shown in fig. 12. An ophiuroid at rest in the position $a$, with two rays against the wall of the dish nearest the window was inverted and placed at $b$. Instead of righting itself toward the window as would be expected if the stimulus received from the contact with the wall were operating, it righting away from the window, using the two rays marked and then moved to the position $d$.

When, however, as is shown in fig. 13, the side toward the window is darkened, with an opaque screen an ophiuroid resting at $a$, when inverted and placed at $b$, will right itself toward the darkened side.

DIRECTION OF LOCOMOTION WITH INTENSE LIGHT COMING FROM ONE DIRECTION

In all the experiments described above no especial attention was paid to the lighting. The ophiuroids were exposed to light rays coming from many directions, and although the experimenting dish was placed in front of a window, the specimens were not subjected to great differences in light intensity. As a result, the reacbon to light was not nearly so pronounced as in tests made in a box painted dead black inside and open only at one end. Such a tox was placed with the open end directed toward the very intense light reflected from a bank of white sand; inside of it was put a rectangular dish, lined with dead black paper and filled with sea-water. Under such conditions when an ophiuroid was placed 
in the dish it almost immediately and almost invariably moved to the darkened end of the dish. Previous stimuli produced by contact with solid walls and methods of handling seemed to have no effect. The experiment was repeated many times, varying the position of the rays with reference to the open end of the box, and almost always the reaction was negative to the light. Usually after a great many trials the ophiuroids showed signs of fatigue, and then might fail to react or even might move in a positive direction. However, the tests showed plainly that the ophiuroids experimented with as a rule react strongly in a negative manner to intense light.

\section{RIGHTING WHEN EXPOSED TO INTENSE LIGHT FROM ONE} DIRECIION

When a normal Ophiocoma riisei is placed with its oral side uppermost in the apparatus just described, it rights itself immediately and quickly. A series of trials made under these conditions showed that the ophiuroids righted almost invariably away from the light. The position of the rays was varied in these tests and also the manner of handling, but the behavior in almost every case resulted in a righting toward the darker end of the dish. While the righting was not always directly away from the lighted end, yet it was evident that the impulse was to right away from the light. After the specimen had regained its normal attitude with the oral side down, it moved almost invariably to the dark end of the dish.

It is of considerable interest to compare the behavior of this ophiuroid with that of the starfish, Echinaster crassispina. We have seen that the ophiuroid reacts negatively to bright light; that is, it rights itself away from the source of the light and then also, after regaining its normal position, continues to move away from the source. On the other hand, Echinaster crassispina, which reacts positively to bright light when moving under normal conditions, rights itself in the same manner as the ophiuroid, i.e., away from the light. So we see the ophiuroid on being inverted rights itself away from the light and then continues to move away 
from the light, while the starfish Echinaster crassispina under similar conditions, also rights itself away from the light, but then moves towards the light. In other words, the intense daylight coming from one direction produces a negative reaction when it strikes the aboral surface of the ophiuroid and also a negative reaction when it shines on the oral surface; on the other hand, this same intensity of light brings about a positive reaction when it stimulates the aboral surface of the starfish, Echinaster, and a negative reaction when it stimulates the oral surface. It appears then that the same intensity of light acting on the oral and aboral surfaces of Echinaster brings about reactions of opposite signs.

\section{INFLUENCE OF VERTICAL DARK AND LIGHT WALLS}

All of the most recent workers in the field of light reactions have recognized the importance of the influence of the reflection of light rays from various surfaces in the region of the organism experimented upon. Bohn in some of his earlier papers and in his paper on the reaction of starfish (1908), and also Mast (1907) and Cole (1907), in their very careful work, have laid special stress on the necessity of taking this factor into account. Both Jennings (1907) and Bohn (1908) find that the direction of movement of a starfish, which ordinarily reacts negatively to bright light, is influenced by vertical dark walls even when they cast no shadow; that is, these starfish when not too far away from the dark wall will react by moving toward it. Bohn also shows that other starfish which react positively to bright light move toward a light colored wall rather than a dark one. Another point on which Bohn (1908) lays stress is that shadows cast on the horizontal surface upon which the starfish lies have but little effect in determining the behavior of the creatures. While at present I can offer no quantitative proof of the correctness of this view for ophiuroids, yet my experiments with hanging dark screens indicate that it holds good. 
EFFECT OF HANDLING ON THE METHOD OF RIGHTING

It is generally believed that taking hold of the ray of a starfish or an ophiuroid has an effect on its subsequent behavior. I thought it worth while to make a series of tests with ophiuroids to determine the effect of handling on the method of righting. The apparatus used for these trials was a tight, deep, wooden bucket, into which no light entered except from above, and even this light was reduced to a minimum by partial covering. Several specimens of Ophiocoma riisei were tested. An ophiuroid was picked up by one ray, inverted and placed at the bottom of the bucket, which of course contained sea-water. The rays on which the ophiuroid righted itself were noted and then the specimen was allowed to rest for several minutes. Taking hold of the same ray the experiment was repeated five times; then another series of five trials were made, taking hold of another ray. In this way twenty-five trials were made taking hold of a different ray after every five trials. Although the light coming in from above probably was not a determining factor in the experiment, yet in order to make sure it had no influence, the position of the ophiuroid was varied when inverted, so that the handled ray was directed in a different direction during each of the five trials, the whole five covering the circumference of a circle.

The results of these trials were very conclusive. Almost invariably the ray handled was not used in the righting, although sometimes an adjacent ray took part in the reaction. The tendency to right away from the stimulated ray was very evident. However, if unhealthy ophiuroids are used, or if fatigue is produced by too many trials closely following one another, the results may be different. Similar tests made with the starfish Echinaster crassispina showed a like tendency to right away from the ray handled, but here the results were not so invariable, in fact, one specimen was found in which the ray handled was used to right upon, and this same behavior continued even when the handled ray was changed. 


\section{SUMMARY}

1. Generally, in locomotion, one ray is not used as a director more than another.

2. The tube-feet may act as temporary suckers and thus may enable the ophiuroid to climb vertical walls.

3. The tube-feet react to food and may carry this food to the proximal end of the ray even when the latter is severed from the rest of the animal. Inorganic objects are not handled in this way.

4. Experiments show that the twisting of the rays at the proximal end is an important factor in righting.

5. The ophiuroids studied show that the contact of a ray with a solid wall is often an important factor in determining the direction of locomotion and that the effect of the stimulus produced is retained for some time.

6. This so-called "memory of past stimulation" seems to be lost when the ophiuroid comes under the influence of a stronger stimulus, such as bright sunlight, coming from one general direction.

7. Under the latter conditions the direction of locomotion is almost always negative and quite definite. It is, however, not stereotyped.

8. The ophiuroids studied right themselves away from bright light and then continue to move away from it; on the other hand, the starfish studied rights itself away from the bright light and then moves toward it.

9. The contact of a ray or rays with a solid wall before the specimen is inverted often has some effect on the direction of righting.

10. A shadow cast on the floor on which an ophiuroid is moving does not seem to act as so strong a stimulus as the shadow produced in a cavity.

11. Ophiuroids undoubtedly react to dark vertical walls even when they cast no shadow.

12. The method of handling an ophiuroid often determines the direction of locomotion and righting. 


\section{BIBLIOGRAPHY}

BoHs, G. 1908 Introduction à la psychologie des animaux à symétrie rayonnée. Bul. Inst. Gen. Psychol.

COLE, L. J. 1907 An experimental study of the image-forming powers of various types of eyes. Proc. Am. Acad. Arts and Sci., vol. 42, no. 16.

1910 Direction of locomotion of the starfish (Asterias forbesii). Science, N. S., vol. 31, no. 795.

Cunnot, L. 1891 Etudes morphologiques sur les échinodermes. Arch. de Biol. T. 11.

Glaser, O. C. 1907 Movement and problem solving in Ophiura. Jour. of Exp. Zoöl., vol. 4.

Grave, C. 1900 Ophiura brevispina. Memoir of the National Academy of Sciences, Washington, D. C.

Hamans, O. 1900 Bronn's Klassen und Ordnungen des Thier-Reichs. Zweiter Band.

Jennings, H. S. 1907 Behavior of the starfish Asterias forreri de Loriol. University of California Publications in Zoölogy, vol, 4, no. 2.

Lows, J. 1900 Comparative physiology of the brain and comparative psychology. G. P. Putnam's Sons, New York.

MAST, S. O. 1907 Light reactions in lower organisms. II. Volvox. Jour. Comp. Neur. Psych., vol. 17, no. 2.

NAGEL, W. A. 1894 Vergleichend physiologische und anatomische Untersuchungen über d. Geruchs- und Geschmackssinn und ihre Organe. Zoologica, Heft. 18.

Österaren, von HJ. 1904 Ueber die Funktion der Füsschen bei den Schlangensternen. Biol. Cent., vol. 24.

Preyer, W. 1886-1887 Ueber die Bewegungen der Seesterne. Mitt. aus der Zool. Sta. zu Neapel, Band 7, 1886-1887.

Romanes, G. J. And Ewart, J. C. 1881 Observations on the locomotor system of Echinodermata. Phil. Trans. Roy. Soc., London, vol. 172, pt. 3.

Romanes, G. J. 1884 Observations on the physiology of Echinodermata. Journ. Linn. Soc., London, vol. 17. 1885 Jelly-fish, star-fish, and sea-urchins. London.

UnxkÜLL, J. voN 1905 Studien über die Tonus. II. Die Bewegungen der Schlangensterne. Zeit. f. Biol. vol. 46. 